\title{
A regression analysis of gene expression in ES cells reveals two gene classes that are significantly different in epigenetic patterns
}

\author{
Sung-Joon Park", Kenta Nakai \\ From The Ninth Asia Pacific Bioinformatics Conference (APBC 2011) \\ Inchon, Korea. 11-14 January 2011
}

\begin{abstract}
Background: To understand the gene regulatory system that governs the self-renewal and pluripotency of embryonic stem cells (ESCs) is an important step for promoting regenerative medicine. In it, the role of several core transcription factors (TFs), such as Oct4, Sox2 and Nanog, has been intensively investigated, details of their involvement in the genome-wide gene regulation are still not well clarified.
\end{abstract}

Methods: We constructed a predictive model of genome-wide gene expression in mouse ESCs from publicly available ChIP-seq data of 12 core TFs. The tag sequences were remapped on the genome by various alignment tools. Then, the binding density of each TF is calculated from the genome-wide bona fide TF binding sites. The TFbinding data was combined with the data of several epigenetic states (DNA methylation, several histone modifications, and $\mathrm{CpG}$ island) of promoter regions. These data as well as the ordinary peak intensity data were used as predictors of a simple linear regression model that predicts absolute gene expression. We also developed a pipeline for analyzing the effects of predictors and their interactions.

Results: Through our analysis, we identified two classes of genes that are either well explained or inefficiently explained by our model. The latter class seems to be genes that are not directly regulated by the core TFs. The regulatory regions of these gene classes show apparently distinct patterns of DNA methylation, histone modifications, existence of $\mathrm{CpG}$ islands, and gene ontology terms, suggesting the relative importance of epigenetic effects. Furthermore, we identified statistically significant TF interactions correlated with the epigenetic modification patterns.

Conclusions: Here, we proposed an improved prediction method in explaining the ESC-specific gene expression. Our study implies that the majority of genes are more or less directly regulated by the core TFs. In addition, our result is consistent with the general idea of relative importance of epigenetic effects in ESCs.

\section{Background}

Embryonic stem cells (ESCs) derived from blastocysts are self-renewal and pluripotent [1-3]. To understand the gene regulatory system in ESCs is an important step for uncovering the process of cell fate determination and for promoting regenerative medicine. Considerable recent evidence indicates that several transcription

\footnotetext{
* Correspondence: park@hgc.jp

Human Genome Center, Institute of Medical Science, University of Tokyo, Japan

Full list of author information is available at the end of the article
}

factors (TFs), so-called core TFs, are indispensable to maintain the pluripotency $[4,5]$. Some of the core TFs reprogram somatic cells back to pluripotent states $[6,7]$. These observations suggest that the regulatory network of TFs apparently governs the self-renewal and pluripotency $[8,9]$. On the other hand, many studies have reported that other TFs can functionally substitute for the core TFs [10-13], suggesting that there still exist additional or alternative TFs unrevealed in the network. Epigenetic modifications are also essential for ESCs

\section{Ciomed Central}


$[14,15]$. Their involvement in the maintenance of the pluripotency is still not well clarified.

To understand the regulatory mechanism underlying in ESCs, a number of methods have been developed. In particular, massive parallel sequencing $[9,16-19]$ and various in silico approaches $[8,9,20,21]$ have yielded comprehensive recent advances in our understanding. In this study, we focus on predicting the gene expression in ESCs with the massive parallel sequencing data. Although a previous study successfully applied a regression model to the prediction [21], the model is based on a generalized weighting scheme to prepare predictors (explanatory variables). Intuitively, such weighting scheme cannot reflect the nature of the spatial rearrangement of TF-binding.

Here, we propose a density-based approach that uses the genome-wide bona fide TF binding sites. First, a publicly available ChIP-seq data [9] is reanalyzed. Then, density profiles of TFs estimated from the ChIP-seq data are adopted as predictors in a simple linear regression model to predict the genome-wide gene expression. Predictors are also combined with epigenetic data, such as H3K4me3, H3K27me3, DNA methylation, and CpG island $[16,17]$. Furthermore, we analyze the regulatory effects of TFs, epigenetic states, and their higher-order interactions by using a pipeline developed in house. We demonstrate the predictive power of the density-based regression model and discuss our findings.

\section{Results}

\section{ChIP-seq data is reproduced and extended}

To minimize artifacts, we refined the binding signals of 12 core TFs in mouse ESC publicly available [9] (see methods). The ChIP-seq peak datasets generated by various tools are hereafter denoted as FP4_Bowtie,
FP4_MAQ, and FP4_Soap2. Also, tag positions mapped by Eland [9] are used for the peak detection (FP4_Eland), and the peak data of Chen et al. is involved (Chen Eland). Thus, we prepared five peak datasets in total.

Differences in numbers and positions between the remapped data and the original data were investigated. As a result, relatively larger number of uniquely mapped tags and peaks were gained compared to the original data (Table S1-S3 in Additional file 1). In regard to peaks (Table 1), FP4 with the previously mapped tags (FP4_Eland) covers $85-98 \%$ of Chen_Eland, and the intensity of overlapped peaks is strongly correlated. Thus, it is deemed that FP4 has reproduced Chen_Eland and extended it with novel peaks in different genomic locations. In contrast, FP4 with remapped tags shows relatively lower reproducibility, whereas peak intensities are still correlated with Chen_Eland except Esrrb (Figure 1B). Similar observations can be found from an independent study [22].

The reason why the numbers vary is twofold. First, algorithmic differences in alignment tools cause the different numbers, particularly due to the gapped or ungapped alignment and random indel for mismatches. Second, thresholds for the peak intensity to distinguish experimental noise are different (Table S4 in Additional file 1). That is, Chen et al. used qPCR refinement with small number of peaks, whereas we used Monte Carlo simulation on each chromosome.

\section{Remapped peaks improve the prediction of gene expression}

To assess the importance of TF bindings, Ouyang et al. [21] successfully applied a regression model to the prediction of absolute gene expression in mouse ESC. We first recover this study. Ouyang et al. used TF

Table 1 Reproducibility of newly detected peaks

\begin{tabular}{|c|c|c|c|c|c|c|c|c|c|c|c|c|}
\hline & \multicolumn{4}{|c|}{ Fold Change } & \multicolumn{4}{|c|}{ Overlap of Chen Eland (\%) } & \multicolumn{4}{|c|}{ Correlation of Peak Intensity } \\
\hline & Eland & Bowtie & MAQ & Soap2 & Eland & Bowtie & MAQ & Soap2 & Eland & Bowtie & MAQ & Soap2 \\
\hline c-Myc & 1.01 & 3.26 & 2.25 & 3.41 & 95.12 & 78.23 & 77.53 & 79.78 & 1.00 & 0.97 & 0.98 & 0.98 \\
\hline E2f1 & 1.03 & 1.34 & 1.36 & 1.40 & 85.41 & 74.67 & 74.83 & 75.70 & 1.00 & 0.98 & 0.99 & 0.99 \\
\hline Esrrb & 2.88 & 3.12 & 3.29 & 3.93 & 99.10 & 88.62 & 89.01 & 90.22 & 1.00 & 0.82 & 0.83 & 0.83 \\
\hline $\mathrm{Klf} 4$ & 2.30 & 3.56 & 3.54 & 3.83 & 97.00 & 91.66 & 90.90 & 92.48 & 1.00 & 0.94 & 0.95 & 0.95 \\
\hline Nanog & 1.01 & 2.15 & 1.84 & 2.42 & 97.93 & 87.93 & 90.06 & 91.69 & 1.00 & 0.97 & 0.99 & 0.99 \\
\hline n-Myc & 1.86 & 3.24 & 3.59 & 3.60 & 95.39 & 84.22 & 85.71 & 86.15 & 1.00 & 0.97 & 0.97 & 0.97 \\
\hline Oct4 & 2.39 & 6.21 & 6.72 & 6.78 & 96.89 & 84.26 & 84.53 & 87.58 & 1.00 & 0.97 & 0.98 & 0.98 \\
\hline Smad1 & 1.49 & 3.19 & 3.24 & 3.53 & 91.56 & 75.58 & 79.84 & 81.62 & 1.00 & 0.85 & 0.86 & 0.88 \\
\hline Sox2 & 1.82 & 4.23 & 4.59 & 4.65 & 98.37 & 90.34 & 90.57 & 92.93 & 1.00 & 0.96 & 0.98 & 0.98 \\
\hline Stat3 & 1.60 & 8.49 & 4.80 & 8.33 & 97.09 & 80.99 & 81.70 & 84.64 & 1.00 & 0.97 & 0.98 & 0.98 \\
\hline Tcfcp2l1 & 1.04 & 1.73 & 1.54 & 1.85 & 89.05 & 90.80 & 91.13 & 92.73 & 0.99 & 0.98 & 0.99 & 0.99 \\
\hline$Z f x$ & 2.62 & 3.81 & 3.94 & 4.06 & 94.89 & 87.67 & 87.61 & 88.42 & 1.00 & 0.96 & 0.97 & 0.97 \\
\hline
\end{tabular}

Fold change is the ratio of newly detected peak number over the original peak number. Overlaps of the original peaks to the newly detected peaks were investigated with 200-bp window. The overlapped peaks were used to calculate the correlation of peak intensity. 


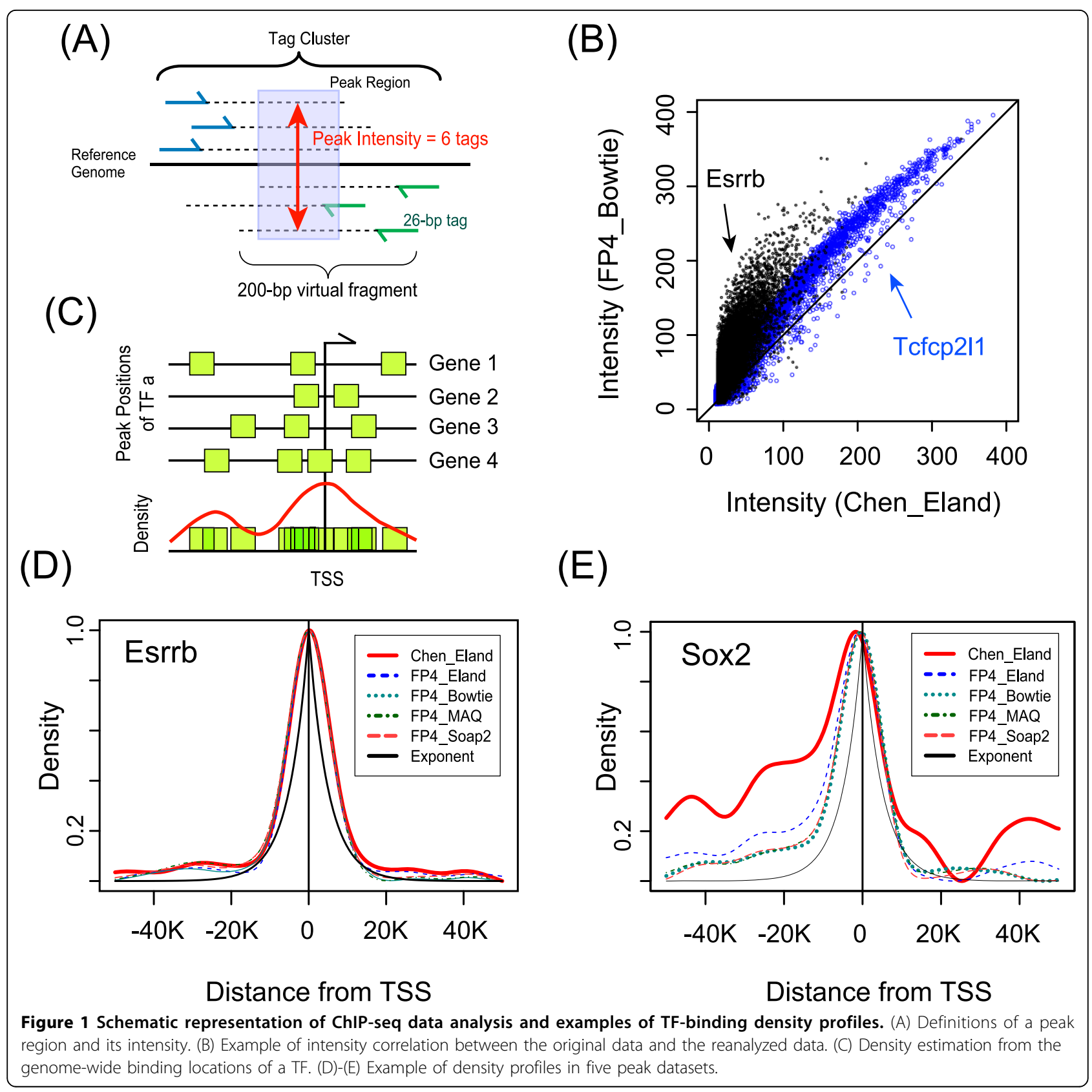

association strength (TFAS) by summing up peak intensities that are weighted exponentially according to the relative positions from TSSs. We applied the TFAS data to our simple linear regression model shown in equation (1), namely exponential-based regression model. The predictive power of our model is much higher $\left(C V-R^{2}=0.647\right)$ than Ouyang's model $\left(C V-R^{2}=0.639\right)$, suggesting that the simple regression model is comparable to their PC-regression model (Figure 2A).

Next, we prepared 17060 genes by removing inconsistency between Ouyang's study and Chen's study. This procedure is prerequisite for gathering precise TF-binding instances. TFAS data for the genes were calculated by the exactly same procedure of Ouyang et al. As a result, the exponential-based model shows $C V-R^{2}=0.495$ with Chen_Eland. In contrast, CV- $R^{2}$ increases to 0.542 (FP4_Eland), 0.587 (FP4_Bowtie), 0.581 (FP4_MAQ), and 0.590 (FP4_Soap2).

These results clearly suggest that the proposed simple linear regression model is applicable to the prediction. Furthermore, it has been demonstrated that the peak datasets we remapped give more information for explaining the gene expression. 


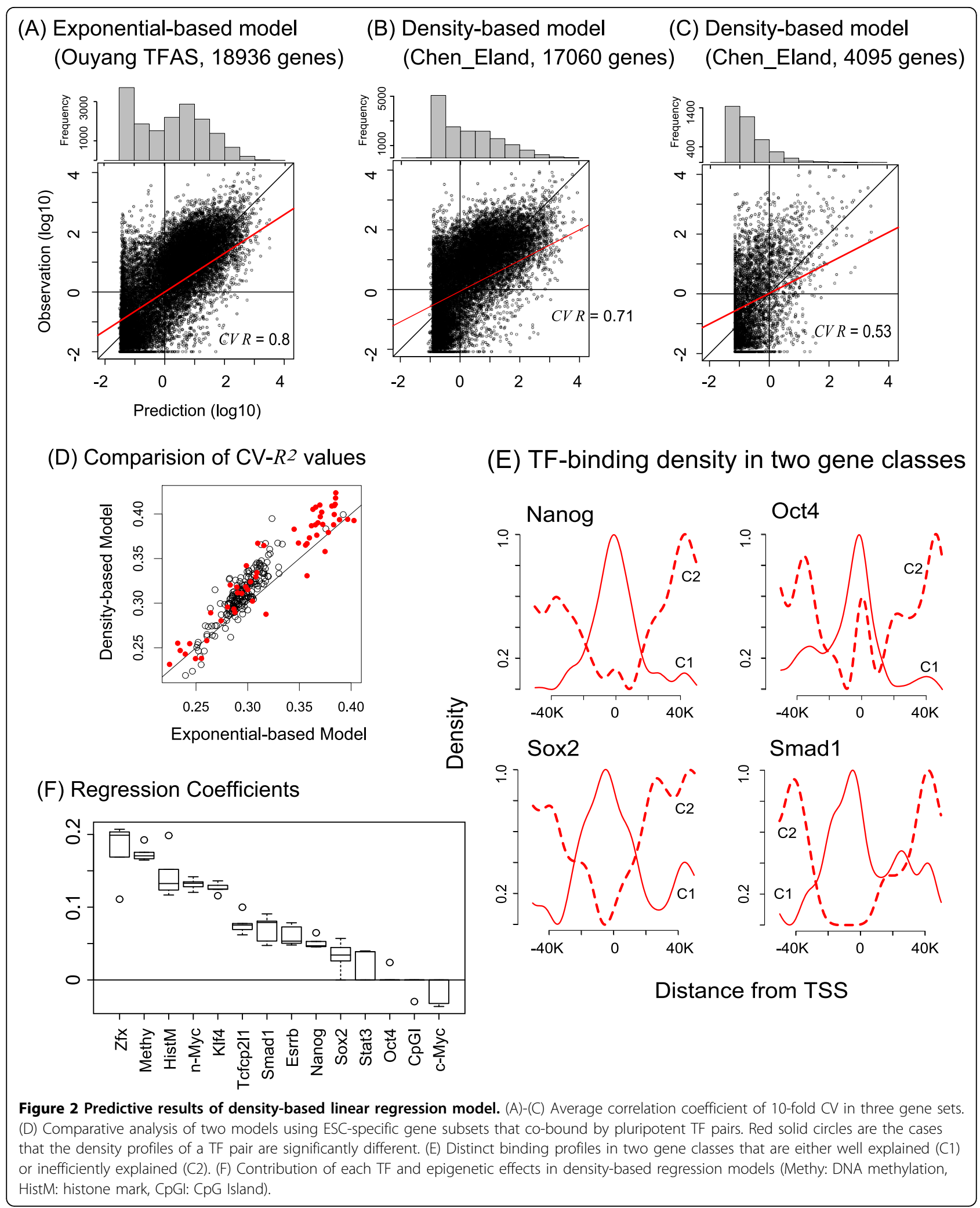




\section{Genome-wide locations of TF bindings do not follow exponential distribution}

To investigate the characteristics of TF binding sites in ESC, the density profiles of TF-bindings are estimated from each of peak datasets (Figure $1 \mathrm{C}$ ), then any two density profiles for a TF in different peak datasets are tested by Kolmogorov-Smirnov (KS) test. According to the KS test, the profiles of a TF are almost identical even if the number of mapped tags and peaks are largely different in, say, Esrrb (Figure 1D). The exceptional case is Sox 2 in Chen_Eland and FP4_Bowtie (Figure 1E) due in part to the stringent filter used in Chen Eland; e.g. loss of Sox 2 peaks in Chen Eland at gene clusters on chromosome X (Figure S1 in Additional file 2).

Importantly, in the same peak dataset, the profiles are significantly different among TFs, e.g. Oct4 and Smad1 in FP4_Bowtie are shown in Figure S2. It is, therefore, thought that spatial preference of TF-bindings cannot be explained by one generalized distribution. In fact, the binding distributions of Nanog, Smad1, Sox2, and Stat3 definitely do not follow the exponential distribution (Figure S2 in Additional file 3).

\section{Density-based regression model outperforms the exponential-based model}

Our observations from the genome-wide distribution of TF binding sites revealed the distinct binding preference from exponential function (Figure 1E). Thus, we use the density profiles as predictors given as equation (2), which we call the density-based regression model. The predictive power of the density-based model with Chen_Eland (Figure 2B) is slightly higher $\left(\mathrm{CV}-R^{2}=0.508\right)$ than the exponential-based model $\left(C V-R^{2}=0.495\right)$. Similar results were obtained when other peak datasets were used.

We suspect that the prediction quality of two regression models may depend on downstream genes that cause specific density profiles. To confirm it, we extracted 4095 ESC-specific genes. E2f1 was excluded here due to its excessive regression coefficient [21]. Then, a subset of 4095 genes that is co-bound by a TF pair was prepared. Since the TFs used are well-known essential regulators in ESCs, the TF pairs, such as Oct4 and Sox2, possibly play an important regulatory role in their downstream ESC-specific genes. All subsets by any combination of two TFs have been prepared.

Figure 2D illustrates that the density-based regression model outperforms in many cases. Furthermore, 55 gene subsets that are co-bound by TF pairs whose density profiles are significantly different $(p<0.05)$ were successfully predicted (red solid circles in Figure 2D). These gene subsets cannot be modeled by a generalized exponential function. The results suggest that the spatial preferences of TF bindings are much more dynamically changed in ESC-specific gene subsets rather than observed from all the genes. This is why the densitybased model improved the predictive power with respect to the generalized exponential-based model.

\section{Two gene classes are different in epigenetic patterns}

It was demonstrated previously that the absolute gene expression in ESCs is predictable by the ChIP-seq data of core TFs [21]. We also confirmed the high predictive power of the regression model. However, the results strongly rely on certain genes whose 'predicted' expressions are constantly lower, but 'observed' expressions are more varied (Figure 2A-C). In Figure 2A, we observed the binomial distribution of predicted expressions that can be partitioned by 1 RPKM (zero on the horizontal axis). We denote $\mathrm{C} 1$ for genes where predicted expression is $\geq 1 \mathrm{RPKM}, \mathrm{C} 2$ for the remains. The conspicuous frequency of $\mathrm{C} 2$ is also observed from Figure 2B-C. C2 genes in Figure 2C consist of 1205 up- and 1254 downregulated genes. Further, the subset of $\mathrm{C} 2\left(\mathrm{C} 22^{\prime}\right)$ where observed expression is greater than 1 RPKM consists of 148 up- and 159 down-regulated genes.

To characterize the gene classes, we analyzed TFbinding profiles and epigenetic modifications. As a result, in $\mathrm{C} 2$ genes, the number of peaks (Figure $\mathrm{S} 3$ in Additional file 2) and density profiles (Figure 2E) are apparently different, implying that the small number of TFs bind to distal regions from TSSs. C2 gene promoters are more methylated (Figure 3A). Remarkably, they tend to be absent from CpG islands (Figure 3B), and be marked with neither $\mathrm{H} 2 \mathrm{~K} 4 \mathrm{me} 3$ nor bivalent domains (Figure 3C). Furthermore, we analyzed gene ontology terms of biological process by DAVID [23]. As a result, $\mathrm{C} 1$ was enriched for positive regulation of gene expression (score $=8.66$ ), whereas C2 was enriched for neural differentiation (score $=34.49)$. C2' was enriched for cell morphogenesis (score $=2.77$ ).

$\mathrm{C} 2$ genes lack the TF-binding instances, implying less direct regulation by the core TFs. This depletion is due in part to excessive non-CpG DNA methylation [16]. Gene ontology analysis shows that $\mathrm{C} 2$ genes are often related to differentiation. Thus, they should be preferentially repressed in ESCs. Interestingly, as the histone marks are relatively rare among $\mathrm{C} 2$ genes, they are likely to be controlled by other regulatory pathways connecting to the maintenance of self-renewal. One possibility is the competitive binding of additional TFs not involved in this study because of the global open chromatin conformation in ESC [19]. Other possibilities include additional epigenetic patterns and homeostatic regulation, further investigations are required. 
(A) DNA methylation

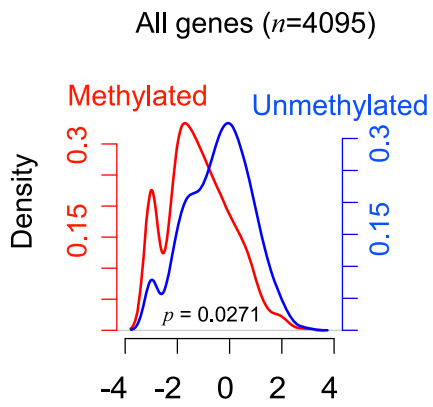

Expression in ESC $(\log 10)$

(B) $\mathrm{CpG}$ Island methylation

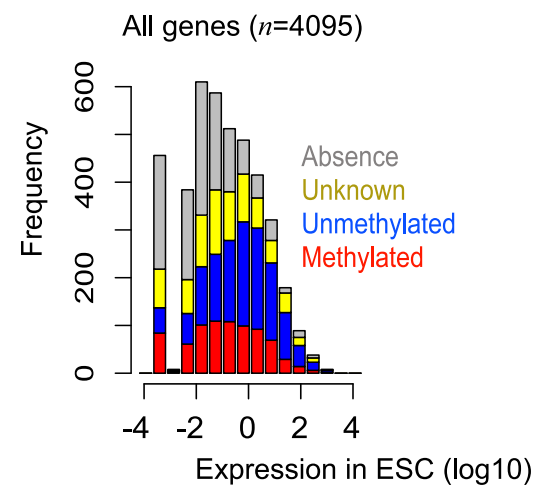

(C) Histone methylation

All genes $(n=4095)$

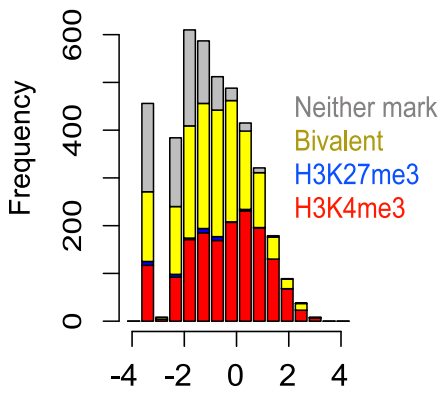

C1 genes $(n=1636)$

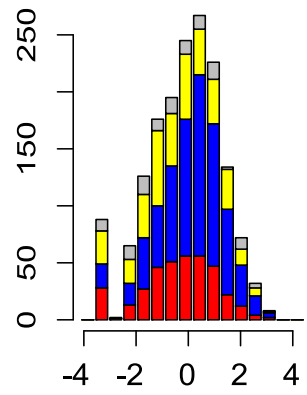

C1 genes $(n=1636)$

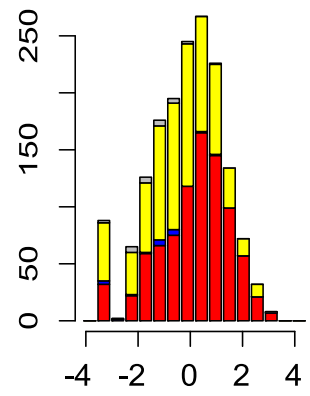

Unmethylated
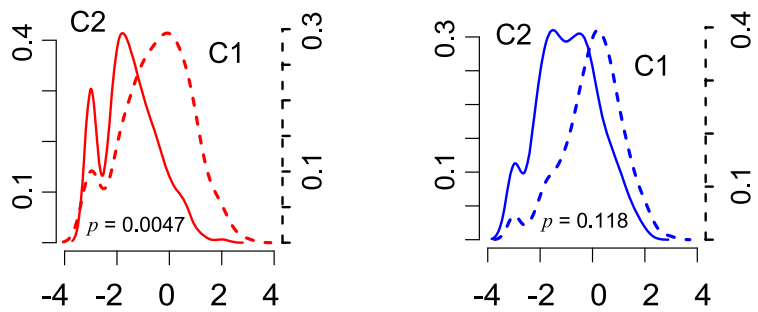

Expression in ESC $(\log 10)$

Figure 3 Epigenetic modifications in ESC-specific genes. Three epigenetic states observed in genes whose expressions are 4-fold up or down in ESC against EB are considered. Gene class $\mathrm{C} 1$ and $\mathrm{C} 2$ are well explained and inefficiently explained genes by the regression analysis, respectively. 


\section{Epigenetic patterns improve the prediction of gene expression}

To further understand the epigenetic effects in gene regulation, we add three epigenetic states to the regression models; histone mark (HistM), DNA methylation (Methy), and CpG island (CpGI). Thus, 14 explanatory variables are used. To identify effective variables in the prediction, we reduced the regression model by using the stepwise model selection. Also, 100 runs of computer simulation that randomly assign the epigenetic states were performed.

All models with the epigenetic effects improved CV- $R^{2}$ with one to three more variables compared with the models without the epigenetic effects (Table 2). The additional variables are the epigenetic effect terms. The results of simulation support that the improvements are not by the chance. In particular, the density-based models with the epigenetic effects are significantly better when remapped peak datasets are used. Furthermore, overall regression coefficients gathered from all the density-based models in Table 2 show the relative importance of epigenetic effects except CpGI (Figure 2F). Note that the positive-biased activities are consistent with the previous study [24].

\section{TF interactions wired with epigenetic effects}

To investigate the cooperative effects among TFs and epigenetic patterns in gene regulation, we exhaustively searched significant interaction terms from our regression model. First, a subset of ESC-specific genes that are co-bound by a specific TF pair is prepared. Then, the saturated model for the genes is constructed. The model involves 469 variables; 14 main effect terms (11 TFs and 3 epigenetic states) and 455 higher-order interaction terms (all the possible pairwise and triplewise interactions). Finally, our pipeline greedily identifies important variables (see methods). This procedure is independently performed with each of five peak datasets.
In total, 215 models were identified in which the predictive power is higher than the models without higherorder terms. These models contained 6-30 variables including at least one interactive term. As an example, the regression model for genes co-bound by Oct 4 and Sox2, a well-known pluripotent complex $[9,25]$, contained 15 terms and improved $C V-R^{2}=0.4126$ from 0.3837 in the model with only 14 main effect terms. This model suggests that 7 interactive terms are important in the explanation of target gene expression. Among them, 3 terms are mediated by the epigenetic effects. The network representation of this model highlights the importance of signaling receptors (Stat3 and Smad1), activating Oct4/Sox2 complex [9] as well as Klf4/CpGI [26], and the interaction of Zfx/Methy newly found here (Figure 4).

With considering the redundancy and conservativity, we represented the interactive terms of 215 models as a network (Figure S4 in Additional file 2). As a result, 19 gene sets covering approximately $86 \%$ of genes (3523 out of 4095 genes) were linked by 28 regulatory edges of the epigenetic effects that are commonly found in the five peak datasets (Figure S4 in Additional file 2). These results suggest that the cooperative interactions between TF and the epigenetic state are indispensable to explain the majority of gene expression in ESCs. In addition, we confirmed that the regression coefficients in Figure $2 \mathrm{~F}$ are dramatically changed in the regression of given gene sets, and also CpGI significantly contributes to the prediction of gene expression (Figure 4).

\section{Discussion}

ESCs are the widely accepted source for the study of many biological principles. Despite recent advances in our understanding of biological systems, the gene regulation in ESCs is only incompletely understood. To explore the regulatory mechanism underlying in ESCs, we constructed a predictive model for explaining the absolute gene expression in mouse ESC. This model

Table 2 Effects of epigenetic patterns in reduced regression models

\begin{tabular}{|c|c|c|c|c|c|c|c|c|}
\hline \multirow[t]{2}{*}{ Model } & \multirow[t]{2}{*}{ Peaks } & \multicolumn{3}{|c|}{11 TFs } & \multicolumn{3}{|c|}{11 TFs +3 epigenetic effects } & \multirow{2}{*}{$\begin{array}{l}\text { Simulation } \\
\text { CV- } R^{2} \\
\end{array}$} \\
\hline & & CV-R & $C V-R^{2}$ & Variables & CV-R & $C V-R^{2}$ & Variables & \\
\hline \multirow[t]{5}{*}{ Exponential } & Chen_Eland & 0.53 & 0.282 & 9 & 0.58 & 0.333 & 12 & 0.283 \\
\hline & FP4_Eland & 0.57 & 0.319 & 10 & 0.59 & 0.351 & 12 & 0.318 \\
\hline & FP4_Bowtie & 0.58 & 0.331 & 8 & 0.60 & 0.356 & 10 & 0.330 \\
\hline & FP4_MAQ & 0.58 & 0.335 & 9 & 0.60 & 0.361 & 12 & 0.334 \\
\hline & FP4_Soap2 & 0.58 & 0.333 & 9 & 0.60 & 0.357 & 11 & 0.331 \\
\hline \multirow[t]{5}{*}{ Density } & Chen Eland & 0.53 & 0.281 & 10 & 0.58 & 0.334 & 12 & 0.282 \\
\hline & FP4_Eland & 0.57 & 0.324 & 10 & 0.60 & 0.358 & 12 & 0.325 \\
\hline & FP4_Bowtie & 0.59 & 0.342 & 9 & 0.61 & 0.366 & 10 & 0.340 \\
\hline & FP4_MAQ & 0.59 & 0.346 & 9 & 0.61 & 0.370 & 10 & 0.345 \\
\hline & FP4_Soap2 & 0.59 & 0.344 & 9 & 0.61 & 0.366 & 12 & 0.342 \\
\hline
\end{tabular}




\section{ESC-specific genes co-bound by Oct4 and Sox2 (FP4_Eland)}
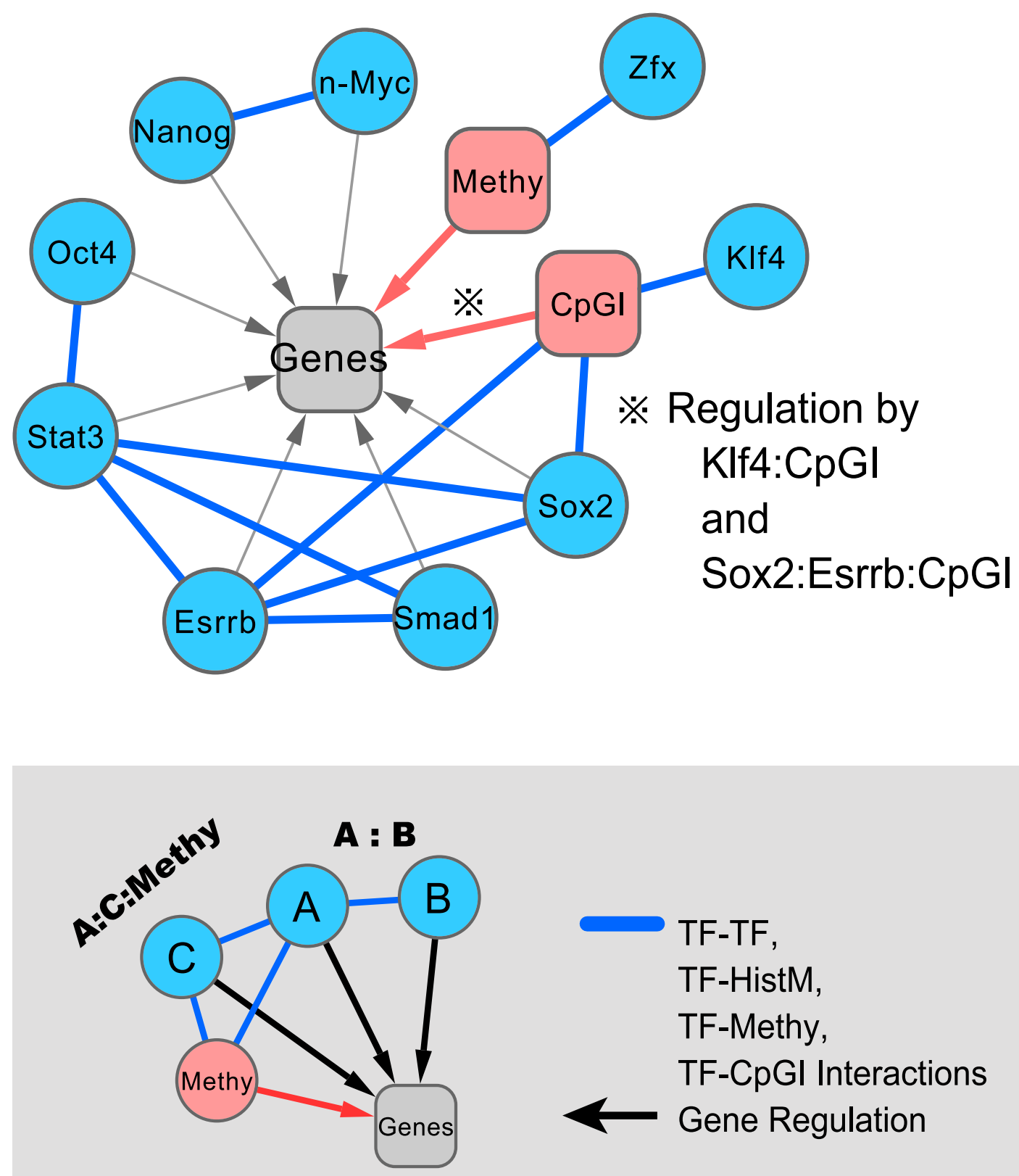

Figure 4 Example of regulatory network of TF interactions with epigenetic effects. This network was generated by the connectivity of nodes in all interaction terms. For example, an interaction term $A: B: C$ is split into three interactions, $A: B, A: C$, and $B: C$. Then, the nodes are linked to each other and to the target gene set. A pairwise interaction with an epigenetic effect is treated differently. For example, in the case of B: Methy, B is not linked to the target gene set. 
uses a novel density-based approach to exploit the recent massive parallel sequencing straightforwardly.

We first reanalyzed the publicly available ChIP-seq data for 12 well-known pluripotent core TFs [9], and retrieved the reproduced and extended TF-binding sites and intensities (Table 1). Using our regression model based on the exponential function [21], we found that the remapped peaks are more informative to explain the gene expression (Table 2). Therefore, we concluded that the algorithmic differences in computer tools for ChIPseq data significantly affect the downstream analysis. Analyzing the heterogeneous peak datasets in a comparative manner, we found that the spatial binding preference of each TF is well conserved in all the datasets, whereas the preferences of TFs in a dataset are significantly different from each other (Figure 1D-E). These results imply that density profiles are better explanatory variables than the generalized exponential function. In fact, the predictive power of density-based model is constantly higher than the exponential-based model (Figure $2 \mathrm{~A}-\mathrm{C}$, Table 2). Even if the density profiles are dynamically changed in certain downstream genes, the proposed model is still outstanding (Figure 2D).

Unexpectedly, we found two gene classes that are either well explained or inefficiently explained by the regression model. The latter class genes have less binding instances of the pluripotent TFs (Figure 2E), possibly related to excessive DNA methylation (Figure 3A). The gene classes show apparently different characteristics in epigenetic modifications (Figure 3), suggesting that they are likely to be under control in different regulatory mechanisms. In the present study, we simply combined the discrete epigenetic states with the powerful densitybased model. This model significantly improved the predictive power (Table 2). Investigating higher-order interactions among the predictors, we found that the cooperative interactions between TF and epigenetic pattern are indispensable for regulating approximately $86 \%$ of ESC-specific genes (Figure S4 in Additional file 2). These results suggest that the relative importance of epigenetic effects to regulate the gene expression in ESCs, supporting the general idea $[14,15]$.

We proposed a powerful regression model, and uncovered the relative importance of epigenetic regulation in ESCs. Overall prediction quality is still insufficient. As future works, comprehensive representation of epigenetic patterns is required, and additional or alternative TFs in ESCs should be considered.

\section{Methods}

\section{Data acquisition}

\section{ChIP-seq data and gene expression}

Raw tag sequences and a control library were downloaded from GEO database (GSE11431). High-quality 26 base pair (bp) tags that have less than three ambiguous bases were mapped to mm8 by Bowtie [27], MAQ [28], and Soap2 [29] with allowing two mismatches. Only uniquely mapped tags were extended to 200-bp virtual fragments (Figure 1A). FP4 (FindPeaks 4.0) [30] detected significant peak regions. Monte Carlo simulation was performed on each chromosome to calculate false discovery rate (FDR). Also, the fold enrichment of tags in each peak region over remapped control tags was measured. Finally, we prepared peaks by criteria, FDR $<5 \%$ and 5 -fold enrichment.

For the absolute gene expression, the number of tags per kilobase of exon region per million mapped tags (RPKM) [18] for 18936 mouse genes in ESC and in embryoid body (EB) were prepared from [21]. Positional information of transcription start sites (TSSs) of 17443 Refseq genes in mm8 were prepared from [9]. Removing inconsistent gene IDs between RPKM data and TSS data, we compiled 17060 genes. We prepared 4095 ESCspecific genes whose expressions are 4-fold up- or down-regulated in ESC over EB. The dataset used in here is available at http://www.hgc.jp/ park/research/.

\section{Epigenetic modifications}

DNA methylation maps are prepared from two datasets that use different high-throughput detection methods [16,17]. Methylation states of high-CpG-density promoters (GC content $\geq 0.55$ ) are defined by mean methylation levels; unmethylated if mean $\leq 0.25$, methylated if mean $\geq 0.75$. The genome-wide distribution of CpG islands and histone mark were downloaded from UCSC genome browser. We consider three histone states; histone $\mathrm{H} 3$ lysine 4 trimethylation (H3K4me3), an active mark of expression, H3 lysine 27 trimethylation (H3K27me3), a repressive mark, and bivalent domain of H3K4me3 and H3K27me3, a 'poised' mark of expression.

\section{Estimation of TF binding density}

Given a genome-wide location map of a TF-bindings, all peak positions were converted to relative positions to the nearest TSSs. Gaussian kernel density function (bandwidth $=300 \mathrm{bps}$ ) estimated the density profile of the TF-bindings within $\pm 50 \mathrm{~K}$ bps. The profile was normalized into range of $[0,1]$ by dividing by the maximal density height.

\section{Regression model}

We use a multivariate regression model

$$
\log Y_{i}=\sum_{j} w_{j} S_{i j}+e_{i}
$$

where $Y_{i}$ is the expression of gene $i, S_{i j}$ is the score of the $j$ th TF on gene $i, w_{j}$ is the regression coefficient of 
the $j$ th TF, and $e_{i}$ is the error term. The score $S_{i j}$ is given by

$$
S_{i j}=\sum_{k} g_{k} F_{j}\left(l_{k}\right),
$$

where $g_{k}$ is the perk intensity of the $k$ th binding peak of the $j$ th TF, $F_{j}$ is the normalized density function for the $j$ th TF, and $l_{k}$ is the relative position of the $k$ th peak to TSS of gene $i$. Note that a small value is added to $Y_{i}$ for the logarithm.

\section{Adding epigenetic effects}

Discrete values representing epigenetic states of a gene $i$ are added to the regression model

$$
\log Y_{i}=\sum_{j} w_{j} S_{i j}+\alpha H_{i}+\beta M_{i}+\gamma C_{i}+e_{i}
$$

where $H$ is the type of histone mark (neither mark $=1.0, \quad H 3 \mathrm{~K} 27 \mathrm{me} 3=2.0$, bivalent $\operatorname{mark}=3.0$, $\mathrm{H} 3 \mathrm{~K} 4 \mathrm{me} 3=4.0$ ), $M$ is the DNA methylation (no annotation $=1.0$, methylation $=2.0$, unmethylation $=3.0), C$ is the CpG island (absence $=1.0$, presence $=2.0$ ), and $\alpha, \beta, \gamma$ are the regression coefficients for $H, M, C$, respectively.

\section{Fitting and reducing regression models}

Explanatory variables in a regression model are logtransformed and quantile-normalized. 10 runs of 10 -fold cross validation $(\mathrm{CV})$ measure the average correlation coefficient $(\mathrm{CV}-\mathrm{R})$ and the average proportion of variation explained by the model $\left(\mathrm{CV}-R^{2}\right)$. The stepwise model selection is done by stepAIC in R language with the backward and forward procedure. The regression model with higher-order interactions are reduced by a pipeline developed in house; ANOVA in R language first diagnoses the significance of each explanatory variable in the given saturated model. Next, significant variables ( $p<0.05$ in F-test) are gathered. Finally, the best model is constructed by adding and removing the collected variables one by one in increasing order of $p$ value until $C V-R^{2}$ is not improved anymore.

\section{Additional material}

Additional file 1: Extended analysis of ChIP-seq data This file provides tables including the summary of tag mapping (Table S1), the fold change of remapped tags over the original data (Table S2), the number of peaks in five datasets (Table S3), and the thresholds used to detect significant peaks (Table S4).

Additional file 2: Comprehensive analysis of gene regulation in mouse ESC This file provides figures including an example of peak distributions (Figure S1), TF-binding instances in two gene classes (Figure S3), and the regulatory network of TFs wired with epigenetic effects (Figure S4)
Additional file 3: Density profile of $\mathbf{1 2}$ TFs This file provides the density profiles of 12 core TFs in five peak datasets (Figure S2).

\section{Acknowledgements}

We thank Dr. Tetsushi Yada (Kyoto University) for helpful discussions. Computational resources were provided by the super computer system at Human Genome Center, Institute of Medical Science, University of Tokyo. This work was supported by the Research Program of Innovative Cell Biology by Innovative Technology (Cell Innovation) by the Ministry of Education, Culture, Sports, Science and Technology-Japan.

This article has been published as part of BMC Bioinformatics Volume 12 Supplement 1, 2011: Selected articles from the Ninth Asia Pacific Bioinformatics Conference (APBC 2011). The full contents of the supplement are available online at http://www.biomedcentral.com/1471-2105/12? issue $=\mathrm{S} 1$.

\section{Competing interests}

The authors declare that they have no competing interests.

Published: 15 February 2011

\section{References}

1. Evans MJ, Kaufman MH: Establishment in culture of pluripotential cells from mouse embryos. Nature 1981, 292(5819):154-6.

2. Martin GR: Isolation of a pluripotent cell line from early mouse embryos cultured in medium conditioned by teratocarcinoma stem cells. Proc Natl Acad Sci USA 1981, 78(12):7634-8.

3. Thomson JA, Itskovitz-Eldor J, Shapiro SS, Waknitz MA, Swiergiel JJ, Marshall VS, Jones JM: Embryonic stem cell lines derived from human blastocysts. Science 1998, 282(5391):1145-7.

4. Greber B, Lehrach $\mathrm{H}$, Adjaye J: Silencing of core transcription factors in human EC cells highlights the importance of autocrine FGF signaling for self-renewal. BMC Dev Biol 2007, 7:46.

5. Silva J, Smith A: Capturing pluripotency. Cell 2008, 132(4):532-6.

6. Takahashi K, Tanabe K, Ohnuki M, Narita M, Ichisaka T, Tomoda K, Yamanaka S: Induction of pluripotent stem cells from adult human fibroblasts by defined factors. Cell 2007, 131(5):861-72.

7. Yu J, Vodyanik MA, Smuga-Otto K, Antosiewicz-Bourget J, Frane JL, Tian S, Nie J, Jonsdottir GA, Ruotti V, Stewart R, Slukvin I, Thomson JA: Induced pluripotent stem cell lines derived from human somatic cells. Science 2007, 318(5858):1917-20

8. Boyer LA, Lee TI, Cole MF, Johnstone SE, Levine SS, Zucker JP, Guenther MG, Kumar RM, Murray HL, Jenner RG, Gifford DK, Melton DA, Jaenisch $\mathrm{R}$, Young RA: Core transcriptional regulatory circuitry in human embryonic stem cells. Cell 2005, 122(6):947-56.

9. Chen $X, X u$ H, Yuan P, Fang F, Huss M, Vega VB, Wong E, Orlov YL, Zhang W, Jiang J, Loh YH, Yeo HC, Yeo ZX, Narang V, Govindarajan KR, Leong B, Shahab A, Ruan Y, Bourque G, Sung WK, Clarke ND, Wei CL, $\mathrm{Ng} \mathrm{HH}$ : Integration of external signaling pathways with the core transcriptional network in embryonic stem cells. Cell 2008, 133(6):1106-17.

10. Yang J, Chai L, Fowles TC, Alipio Z, Xu D, Fink LM, Ward DC, Ma Y: Genome-wide analysis reveals Sall4 to be a major regulator of pluripotency in murine-embryonic stem cells. Proc Natl Acad Sci USA 2008, 105(50):19756-61.

11. Zhang X, Zhang J, Wang T, Esteban MA, Pei D: Esrrb activates Oct4 transcription and sustains self-renewal and pluripotency in embryonic stem cells. J Biol Chem 2008, 283(51):35825-33.

12. Singhal N, Graumann J, Wu G, Arauzo-Bravo MJ, Han DW, Greber B, Gentile L, Mann M, Scholer HR: Chromatin-Remodeling Components of the BAF Complex Facilitate Reprogramming. Cell 2010, 141(6):943-55.

13. Nakagawa M, Takizawa N, Narita M, Ichisaka T, Yamanaka S: Promotion of direct reprogramming by transformation-deficient Myc. Proc Natl Acad Sci USA 2010, 107(32):14152-7.

14. Bernstein $B E$, Meissner $A$, Lander ES: The mammalian epigenome. Cell 2007, 128(4):669-81.

15. Rugg-Gunn PJ, Cox BJ, Ralston A, Rossant J: Distinct histone modifications in stem cell lines and tissue lineages from the early mouse embryo. Proc Natl Acad Sci USA 2010, 107(24):10783-90. 
16. Fouse SD, Shen Y, Pellegrini M, Cole S, Meissner A, Van Neste L, Jaenisch R, Fan G: Promoter $\mathrm{CpG}$ methylation contributes to ES cell gene regulation in parallel with Oct4/Nanog, PcG complex, and histone H3 K4/K27 trimethylation. Cell Stem Cell 2008, 2(2):160-9.

17. Meissner A, Mikkelsen TS, Gu H, Wernig M, Hanna J, Sivachenko A, Zhang X, Bernstein BE, Nusbaum C, Jaffe DB, Gnirke A, Jaenisch R, Lander ES: Genome-scale DNA methylation maps of pluripotent and differentiated cells. Nature 2008, 454(7205):766-70.

18. Mortazavi A, Williams BA, McCue K, Schaeffer L, Wold B: Mapping and quantifying mammalian transcriptomes by RNA-Seq. Nat Methods 2008, 5(7):621-8.

19. Gaspar-Maia A, Alajem A, Polesso F, Sridharan R, Mason MJ, Heidersbach A, Ramalho-Santos J, McManus MT, Plath K, Meshorer E, Ramalho-Santos M: Chd1 regulates open chromatin and pluripotency of embryonic stem cells. Nature 2009, 460(7257):863-8.

20. Nishiyama A, Xin L, Sharov AA, Thomas M, Mowrer G, Meyers E, Piao Y, Mehta S, Yee S, Nakatake Y, Stagg C, Sharova L, Correa-Cerro LS, Bassey U, Hoang H, Kim E, Tapnio R, Qian Y, Dudekula D, Zalzman M, Li M, Falco G, Yang HT, Lee SL, Monti M, Stanghellini I, Islam MN, Nagaraja R, Goldberg I, Wang W, Longo DL, Schlessinger D, Ko MS: Uncovering early response of gene regulatory networks in ESCs by systematic induction of transcription factors. Cell Stem Cell 2009, 5(4):420-33.

21. Ouyang Z, Zhou Q, Wong WH: ChIP-Seq of transcription factors predicts absolute and differential gene expression in embryonic stem cells. Proc Natl Acad Sci USA 2009, 106(51):21521-6.

22. Salmon-Divon M, Dvinge H, Tammoja K, Bertone P: PeakAnalyzer: genomewide annotation of chromatin binding and modification loci. $B M C$ Bioinformatics 2010, 11:415.

23. Huang da W, Sherman BT, Lempicki RA: Systematic and integrative analysis of large gene lists using DAVID bioinformatics resources. Nat Protoc 2009, 4:44-57.

24. Chamberlain SJ, Yee D, Magnuson T: Polycomb repressive complex 2 is dispensable for maintenance of embryonic stem cell pluripotency. Stem Cells 2008, 26(6):1496-505.

25. Chew JL, Loh YH, Zhang W, Chen X, Tam WL, Yeap LS, Li P, Ang YS, Lim B, Robson $\mathrm{P}, \mathrm{Ng} \mathrm{HH}$ : Reciprocal transcriptional regulation of Pou5f1 and Sox2 via the Oct $4 / 50 x 2$ complex in embryonic stem cells. Mol Cell Biol 2005, 25(14):6031-46.

26. Han J, Sachdev PS, Sidhu KS: A Combined Epigenetic and Non-Genetic Approach for Reprogramming Human Somatic Cells. PLOS ONE 2010, 5(8): e12297.

27. Langmead B, Trapnell C, Pop M, Salzberg SL: Ultrafast and memoryefficient alignment of short DNA sequences to the human genome. Genome Biol 2009, 10(3):R25.

28. Li H, Ruan J, Durbin R: Mapping short DNA sequencing reads and calling variants using mapping quality scores. Genome Res 2008, 18(11):1851-8.

29. Li R, Li Y, Kristiansen K, Wang J: SOAP: short oligonucleotide alignment program. Bioinformatics 2008, 24(5):713-4.

30. Fejes AP, Robertson G, Bilenky M, Varhol R, Bainbridge $M$, Jones $S$ J: FindPeaks 3.1: a tool for identifying areas of enrichment from massively parallel short-read sequencing technology. Bioinformatics 2008, 24(15):1729-30.

doi:10.1186/1471-2105-12-S1-S50

Cite this article as: Park and Nakai: A regression analysis of gene expression in ES cells reveals two gene classes that are significantly different in epigenetic patterns. BMC Bioinformatics 2011 12(Suppl 1):S50.

\section{Submit your next manuscript to BioMed Central and take full advantage of:}

- Convenient online submission

- Thorough peer review

- No space constraints or color figure charges

- Immediate publication on acceptance

- Inclusion in PubMed, CAS, Scopus and Google Scholar

- Research which is freely available for redistribution

Submit your manuscript at www.biomedcentral.com/submit
Biomed Central 